\title{
Effect of Alcoholic Extract of Caroob Fruits Ceratonia siliquaon The Physiological and Histological Characteristic of Ovaries of Swiss Rat Ratus ratis
}

\author{
Zainab jawad albu said $^{\mathrm{a}}$ Aseel kamel abdalhussein ${ }^{\mathrm{b}}$ Zeinab yassein abd Aun ${ }^{\mathrm{c}}$ \\ ${ }^{a, b, c}$ College of Girls Education/ Biological department/ University of Kufa.Najaf, Iraq. \\ Zainabj.albusaid@uokufa.edu.iq
}

Submission date:- 17/10/2017 Acceptance date:- 16/4/2018 Publication date:- 29/8/2018

Keywords: Caroob fruit, ovaries, alcoholic extract.

\begin{abstract}
This experiment was designed to evaluate the effect of alcoholic extract of caroob Fruits Ceratonia siliqua on the histological features of female Swiss rat ovaries. The results were showed that high concentration( 300 $\mathrm{mg} / \mathrm{kg}$ ) of alcoholic extract improved the characteristics of ovaries by increasing volumes and numbers of growing follicles primordial, primary and secondary follicles.In addition there were increasing in numbers and volumes of corpus luteum and there were increasing in ovaries weights. Other experimental animals group were treated by lower concentration of of caroob Fruits Ceratoniasiliqua $(100 \mathrm{mg} / \mathrm{kg})$ and microscopically section showed that the ovary cortex contained less numbers of growing follicles and corpus luteum.
\end{abstract}

\section{تأثير المستخلص الكحولي لثمار نبات الخروبCeratoniasiliqua في الصفات الفسلجية والنسجية لمبايض إناث الجرذان السويسرية Ratusratis

اجريت الدراسة في مختبرات كلية التزبية للبنات/ جامعة الكوفة للتقصي عن فعالية مستخلص ثمار نبات الخرنوب Ceratoniasiliqua في

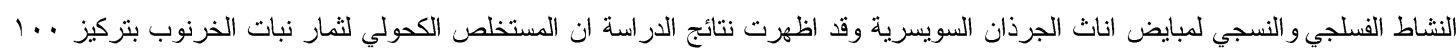

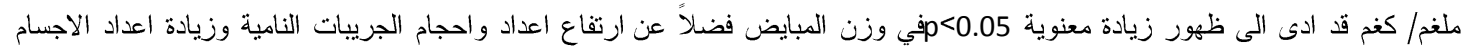

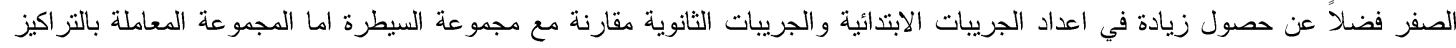

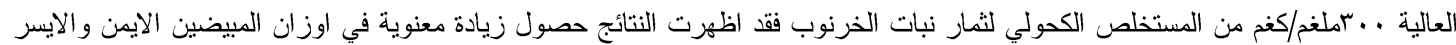

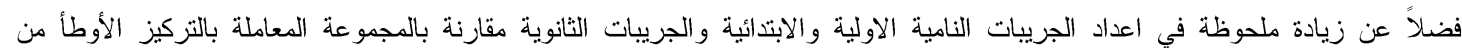

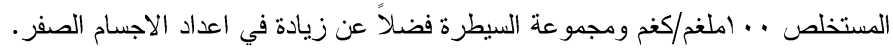
الكلمات الادالة: ثمار الخرنوب، المبايض، المستخلص الكحولي. 


\section{Introduction المقدمة}

تشكل النباتات الطبية وسيلة مستخدمة لعلاج العديد من الامر اض واحدى أهم هذه الامر اض هو مشكلة انخفاض الخصوبة التي تعد واحده

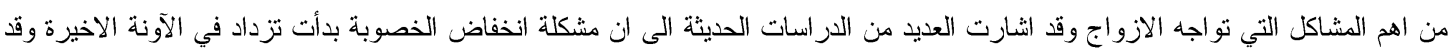

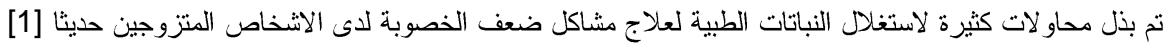

تعد ثمار نبات الخروب او الخرنوب (Ceratoniasilique) واحدة من النباتات ذات الاستخدامات المتعددة في الطب الصيني والطب

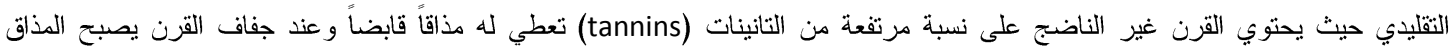

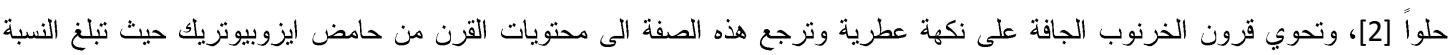

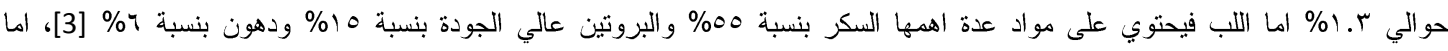

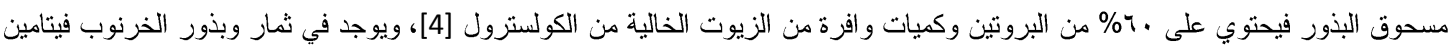

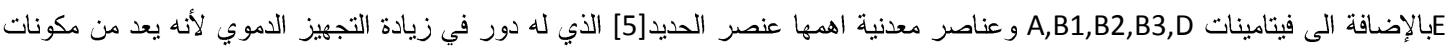

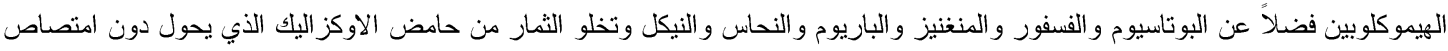

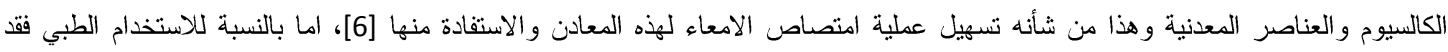

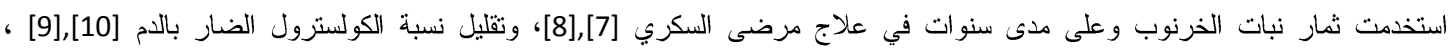

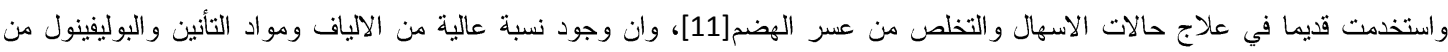

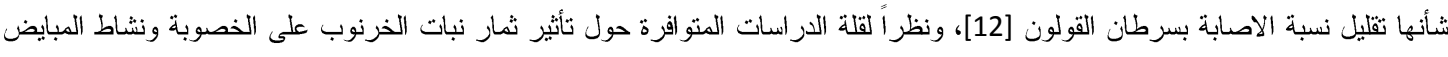
فان الهدف من هذه الدراسة هو تقيم تأثير المستخلص الكحولي لثمار الخرنوب في النشاط الفسلجي والنسيجي لمبايض اناث الجئ الجرذان السويسرية

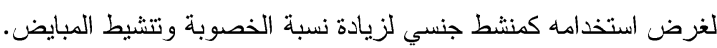

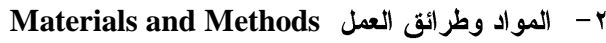

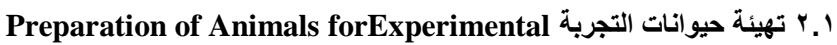
استعملت في التجربة اربع و عشرون انثى جرذوبواقع ثمانية اناث لكل معاملةتراوحت اعمارها بينو-ب أسبو عا وبلغت

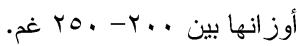
أدخلت الحيو انات في البيت الحيو اني التابع لكلية التربية للبنات/جامعة الكوفة ووضعت في اقفاص بلاستيكية ذات اغطية مشبكة خاصة بها

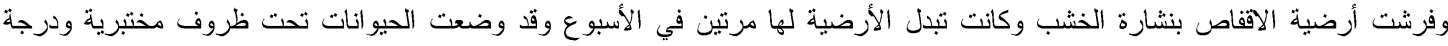

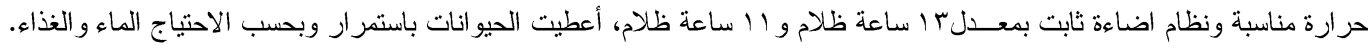

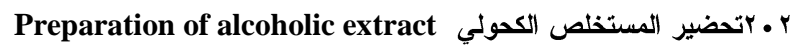

انتعت طريقة [13] وعلى النحو الاتي: وزن ·r غم من مسحوق ثمار الخروب ومزجت مع ..1 مل من كحول الايثانول بتركيز

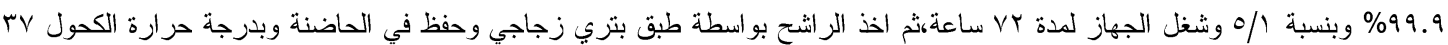
درجة مئوية وترك لحين تبخر الكحول وبعد تبخر الكحول حصل على مستخلص جيلاتيني وبعد ذلك اضيف للمستخلص الجيلاتيني ماء مقطر للحصول على تركيز مخفف من المستخلص الجاهز لحقن الجرذان.

ثم استخدم تركيزين منه لتحضير محلولين مخفقين للمحلول الأصلي ومعاملة حيوانات التجربة بحقنها في الغشاء البريتوني وباستعمال

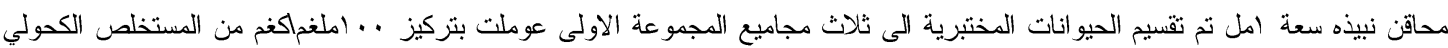

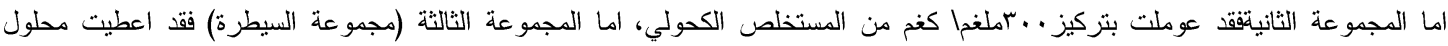
الملح الفسلجي مormal Saline.

Animals Scarification ب. T التضحية بالحيو انات

أجريت التضحية بالحيو انات المعاملة بطريقة التخدير بالكلوروفورم حيث عمل شق طولي ابتداء من المخرج وحتى عظم القص وتم استئصال

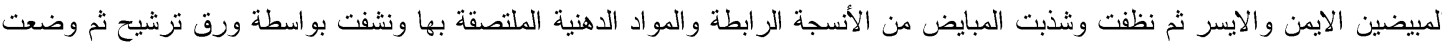
في فورمالين وفي قناني بلاستيكية وعلمت وحفظت في محلول مثبت فورمالين • (\% لحين استعمالها لتحضير الثرائح. 


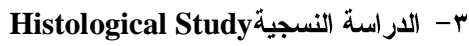

حضرت المقاطع النسجية للأعضاء التكاثرية الأنثوية المتمنلة بالمبيضبن للتعرف على تأثير،المستخلص عليها وقد اتبعت طريقة[14 وكالاتي.

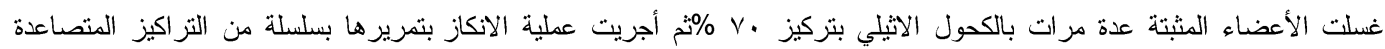
للكحول الاثيلي (100\%_Clearing) بالز ايلين لمدة ساعتين

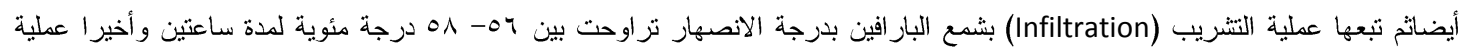

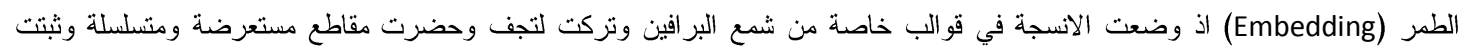

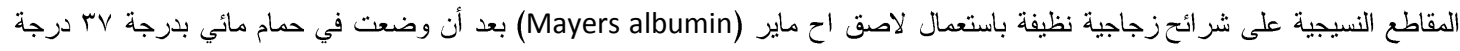

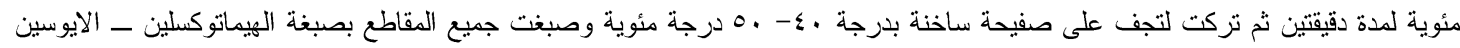

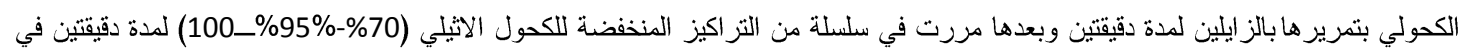

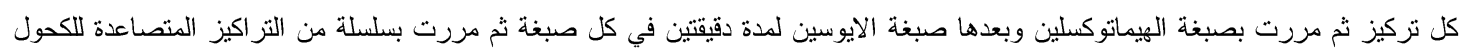

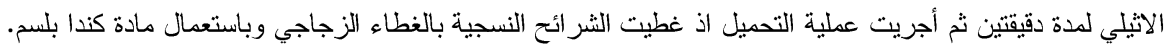

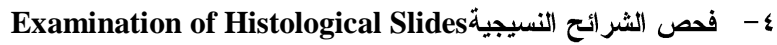

بعد ان حضرت الثرائح النسجية فحصت باستعمال المجهر المركب (CompoundMicroscope) وقد تضمنت الدراسة النسيجية ملاحظة

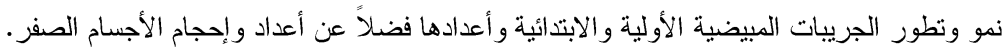

Statistical analysis - - - التحليل الإحصائي عُرضت نتائج الدر اسة على شكل المعدل الخطأ القياسي وحللت أوزان المبايض باستخدام نظام SPSS بمستوى أحتمال0.05> P التقييم نتائج

البحث من الناحية الأحصائية [15] تلثئة

1-تأثير المستخلص الكحولي على معدل وزن المبايض (الأيمن والأيسر)

أظهرت نتائج الجدول (1) حدوث ارتفاع معنوي (P>0.05) في وزن المبيضين الأيمن والأيسر لمجموعة الثانية (T2) التي عوملت

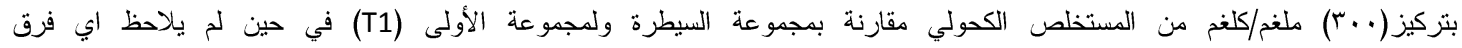
معنوي(P>0.05) في وزن المبيضين الأيمن والأيسر لمجموعة الأولى (T1) ومجموعة عنة السيطرة. جدول (1) تأثير المستخلص الكحولي على معدل وزن المبايض (الأيمن والأيسر) \ الخطأ القياسي

\begin{tabular}{|c|c|c|c|}
\hline المعاملة الثانية(T2 ) & المعاملة الأولى( T1) & السيطرة (C) & المجموعات \\
\hline $0.0017 \pm 0.0681^{b}$ & $0.0065 \pm 0.0532^{a b}$ & $0.0012 \pm 0.0321^{a}$ & اوزان المبايض اليمنى \\
\hline $0.0150 \pm^{b} \quad . v \leqslant r$ & $0.0081 \pm 0.0598^{a b}$ & $0.0031 \pm 0.0326^{a}$ & اوزان المبايض اليسرى \\
\hline
\end{tabular}

* الحروف الصغيرة المختلفة تعني وجود فرق معنوي. " القيم تمثل الوسط الحسابي 土 الخطأ القياسي.

- V تأثير المستخلص الكحولي في الصفات النسجية للمبيضين الايمن والايسر

أظهرت المقاطع النسيجية الصور(T، آ) المأخوذة من المجموعة الثانية (T2) المعاملة بتركيز .. سمغم/كلغم زيادة ملحوظة في أعداد الجريبات المبيضية الناضجة والأولية والابتدائية والثانوية والأجسام الصفر وزيادة التجهيز الدموي للمبيض مقارنة بمجموعة السيطرة الصور (1، (1.0.

بينما أظهرت المقاطع النسجية الصور(r،؛)المأخوذة من المجموعة الأولى (T1) المعاملة بتركيز ·. املغم/كلغ زيادة قليلة في أعداد الجريبات المبيضية الناضجة والأولية و الابتدائية والثانوية والأجسام الصفر مقارنة بمجموعة السيطرة (C) ومجموعة الثانية (T2). 


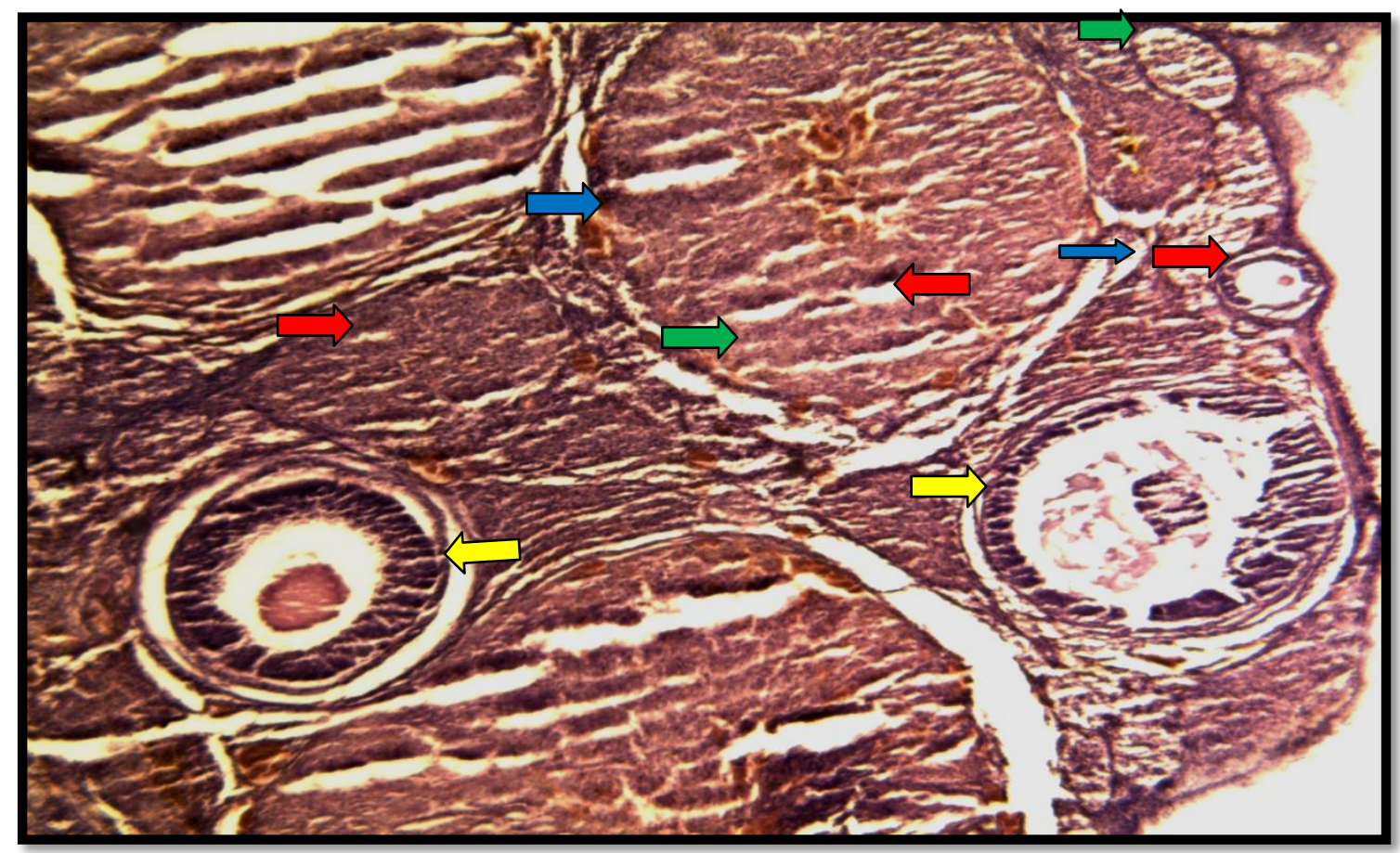

صورة(- 1) توضح مقطع في نسيج المبيض الأيمن لمجموعة السيطرة المعاملة بمحلول الملح الفسلجي, يلاحظ فيها تناقص في نمو وأعداد

الجريبات المبيضية الابتائية (سهم الأحمر),الجريبات الثانوية (سهم الأصفر),الجريبات الأولية (سهم الأخضر) واجسام الصفر (سهر

الأزرق), (هيماتوكسلين -(يوسين(4x).

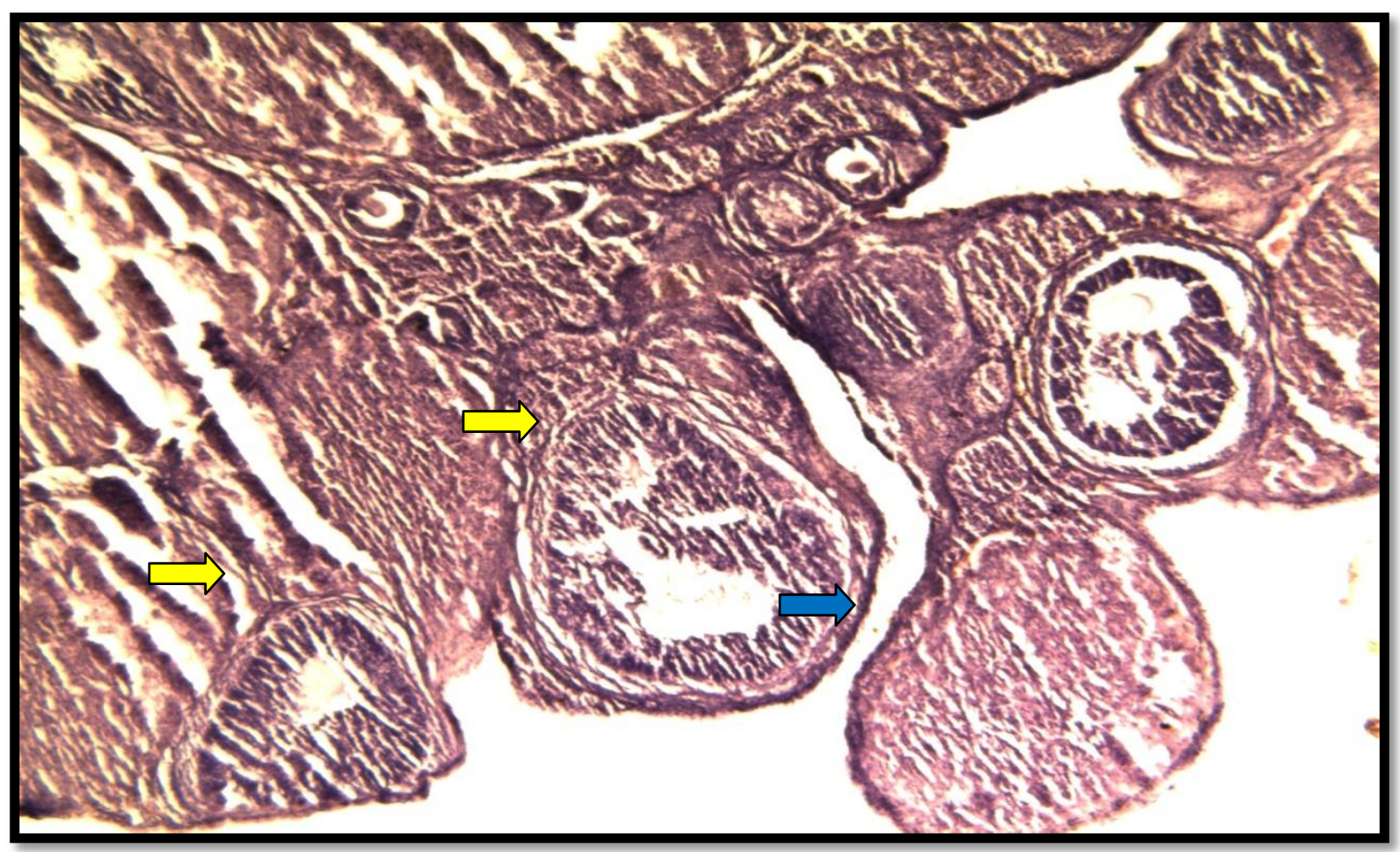

صورة(ץ) توضح مقطع في نسيج المبيض الأيمن لمجموعة الأولى المعامل بتركيز ( . . 1) ملفم/كفم من مستخلص الخرنوب ريلاحظ فيها زيادة في أعداد الجريبات الابتأئة (سهم الأحمر),مقابل التناقص في أعداد الجريبات الثانوية (سهم الأصفر),الجريبات الأولية (سهم الأخضر)

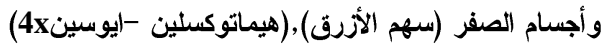




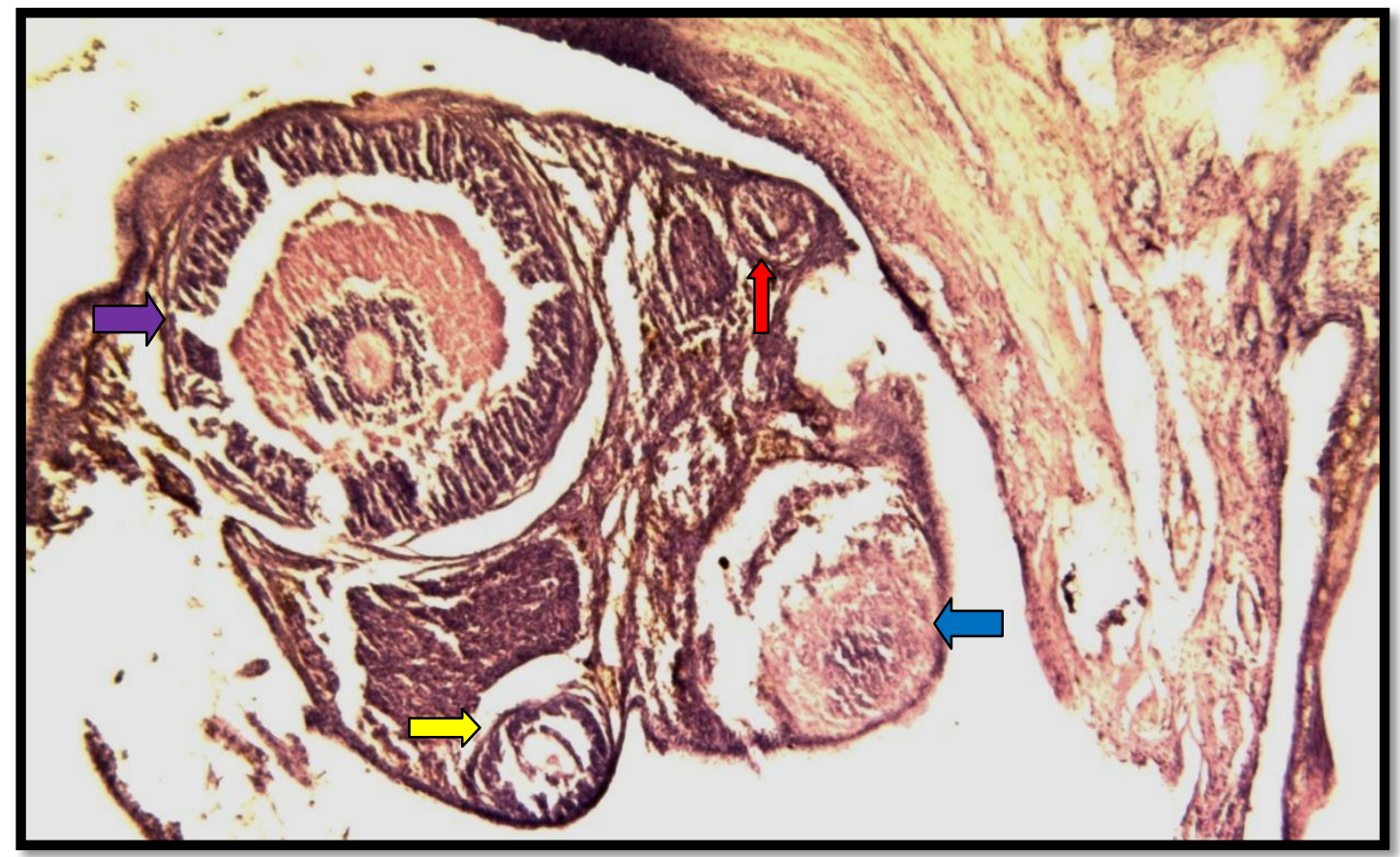

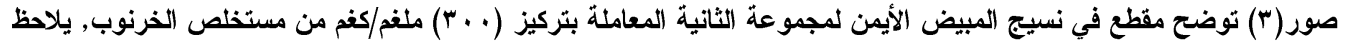
فيها زيادة ملحوظة في أعداد الجريبات المبيضية الناضجة (سهم البنفجي),الجريبات الثانوية (سهم الأصفر),الجريبات الابتائية (سهم الأحمر) وأجسام الصفر (سهم الأزرق), (هيماتوكسلين-ايوسين) (4x).

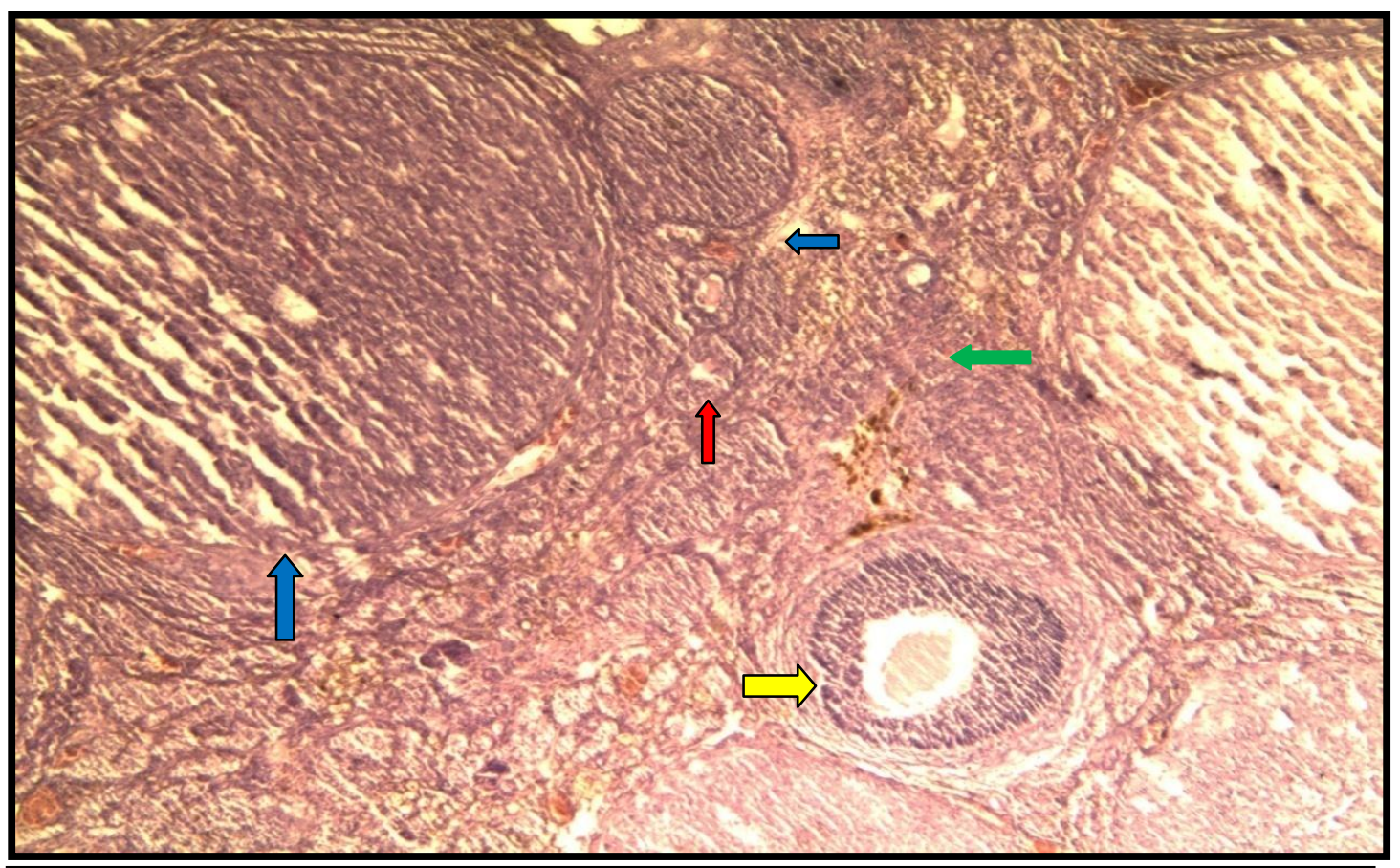

صورة (§) توضح مقطع في نسيج المبيض الأيسر المعاملة بتركيز ( . . 1) ملفم/كفم من مستخلص الخرنوب, يلاحظ فيها زيادة قليلة في

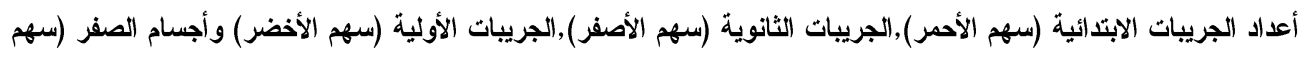
الأزرق), (هيماتوكسلين -ايوسين (4x). 


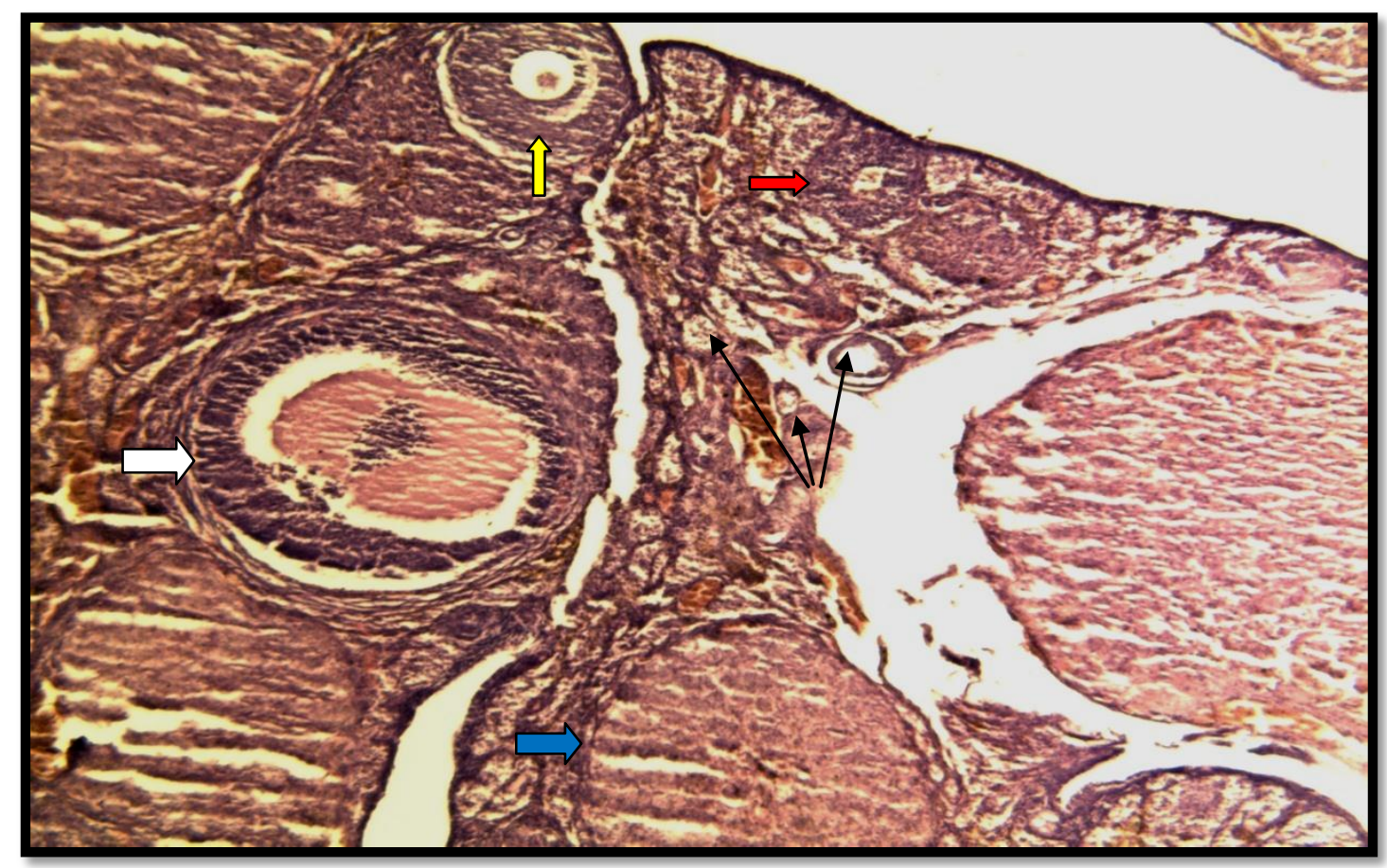

صورة(ه) توضح مقطع في نسيج المبيض الأيسر لمجموعة السيطرة المعاملة بمحلول الملحالفسلجي يلاحظ فيها تناقص في أعداد

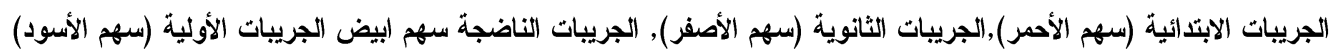

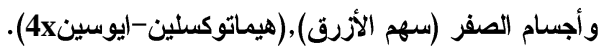

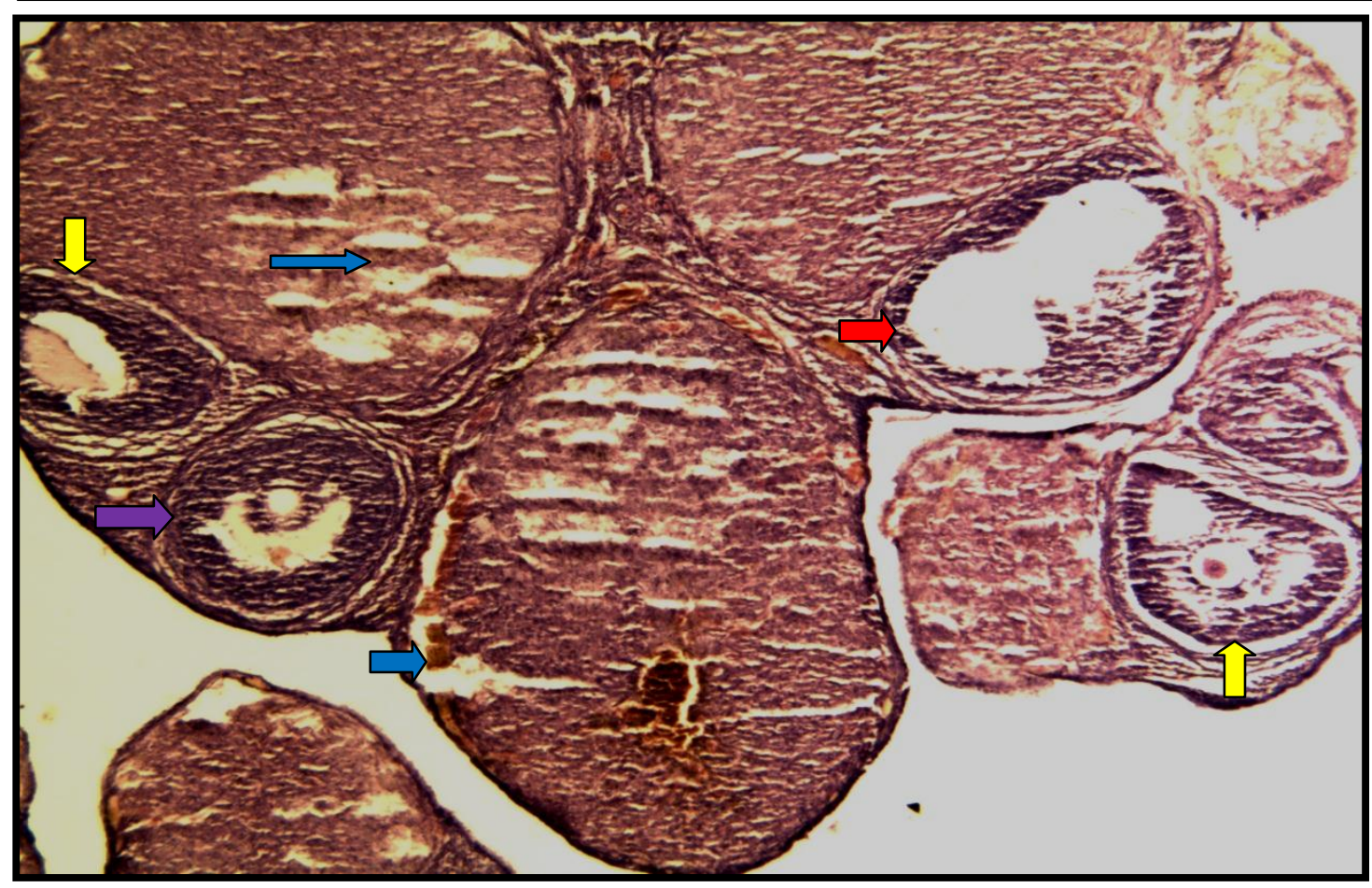

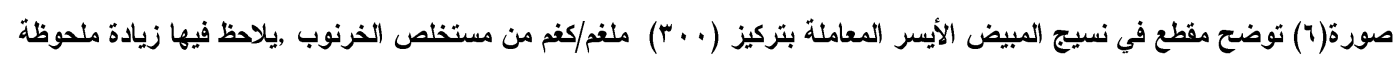
في أعداد ونموالجريبات الناضجة (سهم البنفسج) وكنلك زيادة في أعداد الجريبات الثانوية (سهم الأصفر),وانخفاض عداضد الجريبات

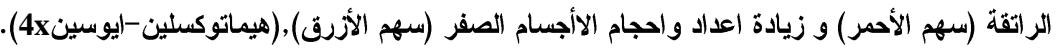


مiscussion المناقشة

لقد أجريت العديد من الدراسات المتعلقة باستخدام النباتات الطبية لتحسين الأداء الجنسي وزيادة معدلات الخصوبة ووفقا للطب التقليدي حيث

من ممكن استخدام الأعشاب لتحسين الخصوبة [16].

وفيما يتعلق بالمستخلص الكحولي لقنور ثمار نبات الخرنوب Ceratoniasiliqua وتأثيره في الصفات الفسلجية والنسيجية للمبايض فأن

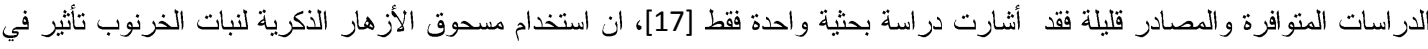

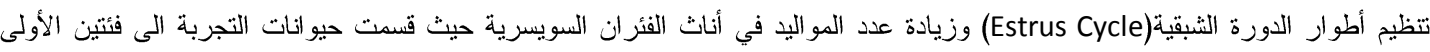
عوملت بالمسحوق الجاف للأزهار الذكرية لنبات الخرنوب بطريقة الاستشاق(Nasal Aspiration) في حين الفئة الثانية تم معاملتها

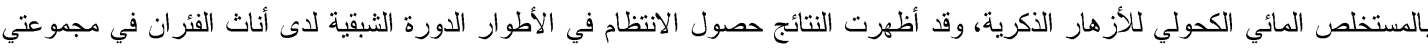
المعاملة مقارنة بمجموعة السيطرة, هذه الدر اسة متفقة الى حذ ما مالى مع نتائج الدر اسة الحالية. حيث أظهرت نتائج الدراسة الحالية زيادة معنوية في أوزان المبايض الأيمن والأيسر جدول (1) في المجموعة المعاملة بالتركيز العالي من

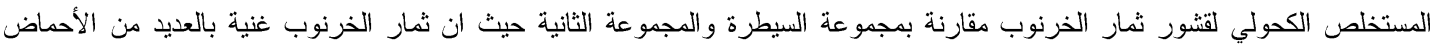

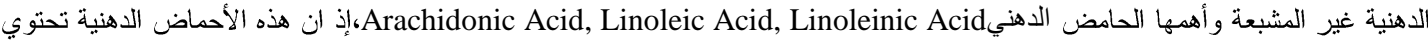
على الاو اصر المزدوجة وهي ضرورية للنمو الطبيعي لأن هذه الأحماض لا يمكن تصنيعها داخل جسم الكائن الحي أويكون تصنيعهاغيركافٍ

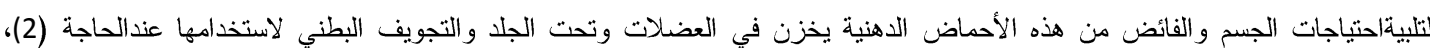
وأظهرت المقاطع النسجية في الدراسة الحالية حصول زيادة ملحوظة في أعداد الجريبات المبيضيةلإناث الجرذان السويسرية.

ولوحظ زيادة نشاط المبايض في المجموعة التي تم معاملتها بالمستخلص الكحولي لنبات الخرنوب بتركيز . .ب ملغم/كغ مقارنة بالمجموعة

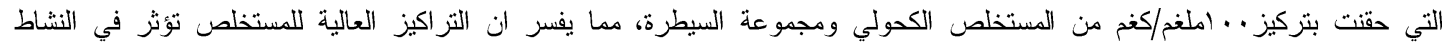

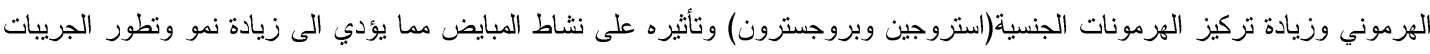

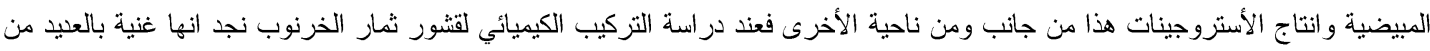

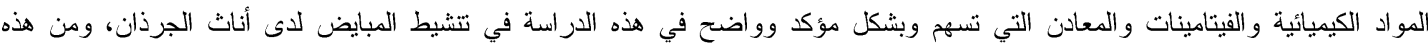

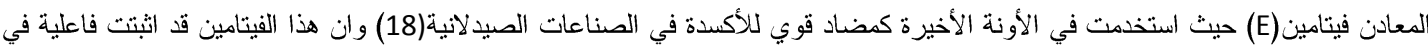
تقليل من تأثير المواد المشعة كعنصر الكادميوم على أنسجة الخصية وتكوين الحيامن في الذكور الفئران مقارنة بالمجموعة غيرة فئر المعاملة بفيتامين

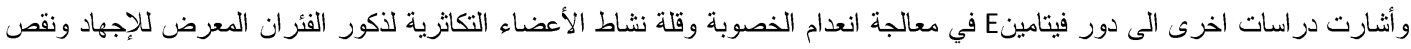
التغذية [20]، فضلاً عن فيتامين فأن ثمار الخرنوب تحتوي على عدد من المركبات الكيميائية التي تعد مركبات وسطية لإنتاج الهرمونات

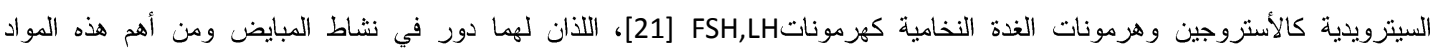

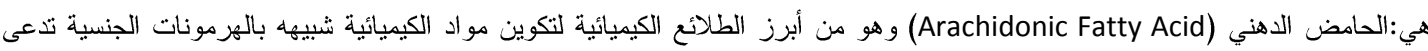

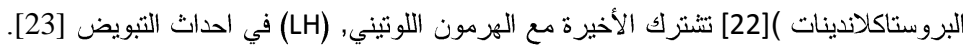
ومن الجدير بالذكر أن الحامض الدهني Arachidonic Acid يوجد بهيئة غير فعالة وبتراكيز قليلة في أغشية الخلايا الحبيية للجريبات

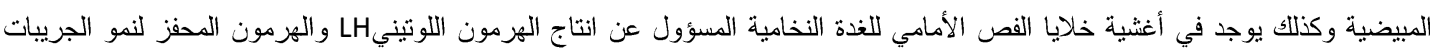

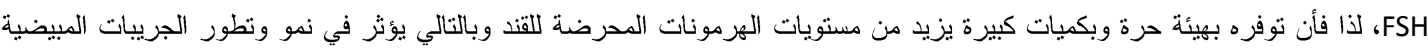

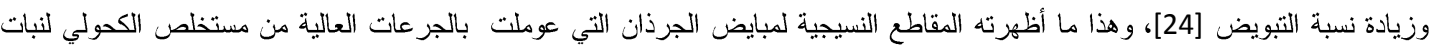

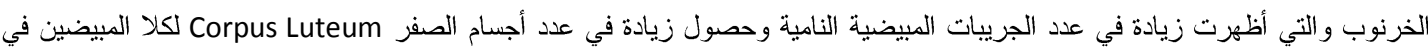

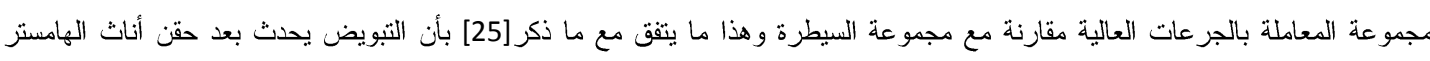

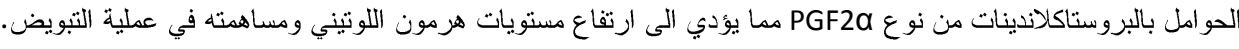

وان عددا من الباحثين منهم [26] اثنار الى دور الهرمون النخاميHابالبروستاكلاندينات في احداث التبويض حيث ذكر الباحث ان البويضة تبقى في شر الك حويصلة كر اف مالم ترتفع مستويات البروستاكلاندينات بموازاة تدقق الهرمون اللهون اللونيني.

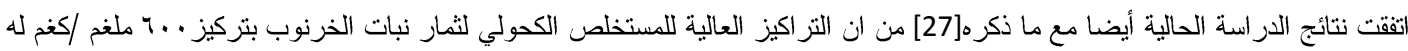

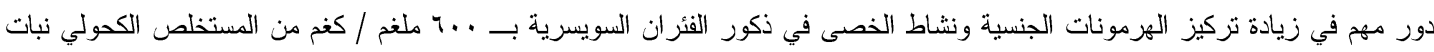

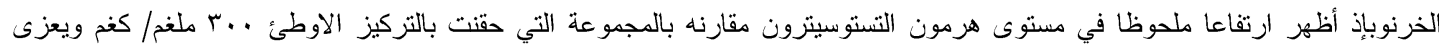

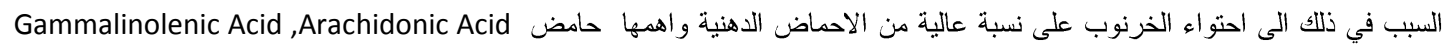
والحامض الت التي لها دور في البناء والتخليق الحيوي للبروستاكلاندينات وهرمون التسترون وبالتالي زيادة في نشاط المبايض 


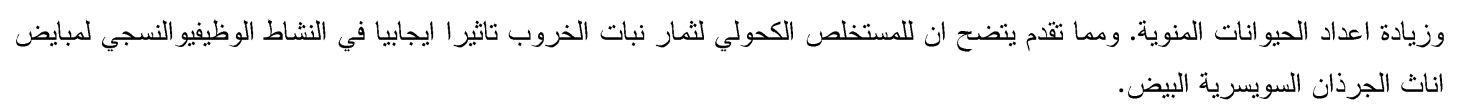

CONFLICT OF INTERESTS

There are no conflicts of interest.

References

1- R.J. Aitken,. "The Amoroso Lecture The human spermatozoon-a cell in crisis?" J ReprodFertil., 115: 1-7. 1999.

2- R.Avallone,M. Olessi, M. Baraldi and A. Monzani. "Determination of chemical composition of carob (Ceratoniasiliqua): Protein, Fat, Carbohydrates, and Tannins", Journal of food composition and Analysis, 10(2): 166-172. 1997

3- F.A. Ayaz, H. Torun, S. Ayaz, P.J. Correia, M. Alaiz, C. Sanz, J. Gruz, M. Strand. "Determination of chemical composition of Anatolian carob pod (Ceratoniasiliqua L.): sugar, Amino and organic Acids, Minerals and phenolic compounds". J Food Qual., 30(6): 1040-1055. 2007

4- A. Haji sharifi. The Encylopedia of medicinal plant. 1st ed, Tehran: Hafezenovin, pp: 384-385. 2008

5-Moh'DKhaiv, J., EL-shatnawi and Khalil I. Ereifej."Chemical Composition and Livestock Ingestion of carob (Ceratoniasiliqua L.)seeds". Journal of Range Management, 54(6): 669-673.2001

6- A. Karabulut, O. Canbolat and A. Kamalak,. Evaluation of carob, Ceratoniasiliqua Pods as a feed for sheep, Department of animal, Nutrition, Kahramanmaras, TURKEY, Livestock Research for Rural Development18 (7). 7 Yaniv, Z., A. Dafni, J. Friedman and D. Palevitch, 1987. plants used for the treatment of diabetes J Ethnopharmacol., 19(2):145-151. 2006.

8- C.A. Edwards, N.A. Blackburn, L. Craigen, P. Davison, J. Tomlin, K. Sugden and et al., "Viscosity of food gums determined in vitro related to their hypoglycemic Actions". Am J ClinNutr, 46(1): 72-77. 1987.

9- H.J. Zunft,W. Luder, A. Harde, B. Haber, H.J. Graubaum and J. Gruenwald. "Carob pulp preparation for treatment of hypercholesterolemia", J AdvTher, 18(5): 230-6. 2001

10- B. Elsenhans, U. Sufke, R. Blume and W.F. Caspary,. The influence of carbohydrate gelling agents on rat intestinal transport of monosaccharides and neutral amino acid in vitro. ClinSci (Lond). 59(5): 373-80. 1980.

11- M.Hostettler, R. Steffen, A. Tschopp,. Efficacy and tolerance of insoluble Carob fraction in the treatment of travelers, diarrhea. J Diarrhoel Dis Res., 13(3): 155-8. 1995.

12- S.Klenow, M. Glei, R. Owen and B. Pool-Zobel. "Carob Fibre compounds modulate parameters of cell growth differently in human HT29 colon adenocarcinoma cell than in LT97 colon adenoma cell". Food and Chemical Toxicology, 46(4):1389-1397. 2008.

13- J.B. Harborne. "Phytochemical MethodChapman and Hull”. New York Is ted. p.p.238. 1973.

14-Bancroft,3.D.and A. stevens,.Theory and practice of histologic al techniques 2thed Williams clews limited beccles and London p.p :116-613. 1982.

15- J.Pallant."SPSS Survival manual; Astep guide to data analysis using SPSSfor windows(version 12)". $2^{\text {nd }}$ edition.printed by ligareSydney.Statistical methods-computer. 2005

16- A. Zargari. "Medicinal Plants”. 2nd ed, University publications, Tehran, Iran, p: 114-116. Persian. 1996.

17-TAber,h.9, W. Bash, N.Taradat, M.sharaf. "Effect of carob (ceratoniasiliqua L.)male flower on the ovulation in mice". 2nd con ference on Biotechnology and Appication Roseareh 26-27 pp:76-77. 2010.

18 - I.M.W. Ebisch, C.M.G. Thomas, W.H. Peters, D.D.M. Braat, R.P.M. Steeger-Theunissen. The importance of folate, Zinc and antioxidants in the pathogenesis and prevention of subfertility.J Hum Repord Update., 13(2): 16374. 2007.

19- H.S. Yang, D.K. Han, J.R. Kim, J.C. Sim. "Effects of alpha-tocopherol on cadmium-induced toxicity in rat testis and spermatogenesis". J Korean Med Sci., 21(3): 445-51. 2006

20- H.Mozdarani, M. Salimi.” Numerical chromosome abnormalities in 8-cell embryos generated from gammairradiated male mice in the absence and presence of VitamineE".Int J Radiat Biol., 82(11): 817-22. 2006.

21-Shadan Farrokh, M.D. "Textbook of Medical Physiology”, vol. 2, Tehran, Ninth Edition, Tchehr CO. pp: 1506. 1996.

22- S.M. karim, "Prostaglandins: chemical and Bio chemical Aspects”. university park press.,u.s. 1976. 
23- H.R. Behrman, G.p. orczk, and R.O. Greep. "Effect of synthesis GnRH on ovulation by Aspirin and Indomethacin". Prostaglandins,1:245.1972.

24- O, Grady ,T.p., B.v. Caldwell, F.Awlett, and H.speroff. "The effect an in hi bittor of prostaglandin synthesis (Indo meth- pseudo pregnancy in the rabbit”. prostaglandins,I:97.1972.

25- A.P.Labhse.twar. Luteolysis and ovulation induced by prostaglandines F2 \{ in the hamster Nature 230. 1971.

26- I.m. Bobak, M. D. Tensen, and M.K. Zalar .Maternity and gynecology care :The Nurse \{the Family the c.v. mosby.com pany, u.sA.1989.

27-Mokhtari M., Ph.D. E.Sharifi, MSc,Sh. Azadian,MSc. The. "Alcoholic extract of ceratoniasilique L. seeds on pituitary-testis hormones and spermatogenesis in rat".Islmic Azad University,Kazerun Branch ,Department Of Bilogy ,Kazerun, Iran.2012. 